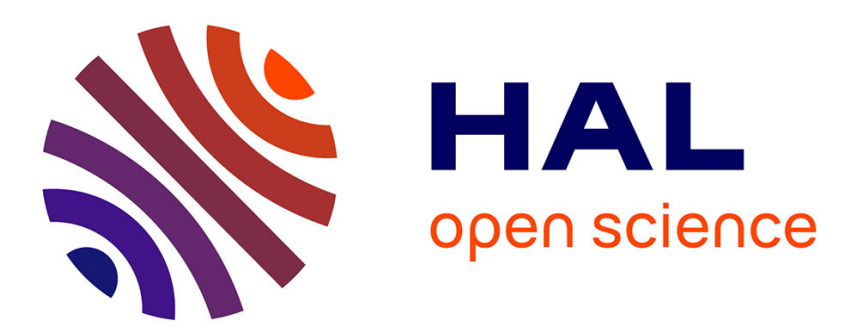

\title{
Ab Initio Simulations of Water Dynamics in Aqueous TMAO Solutions: Temperature and Concentration Effects
}

\author{
Guillaume Stirnemann, Elise Duboué-Dijon, Damien Laage
}

\section{To cite this version:}

Guillaume Stirnemann, Elise Duboué-Dijon, Damien Laage. Ab Initio Simulations of Water Dynamics in Aqueous TMAO Solutions: Temperature and Concentration Effects. Journal of Physical Chemistry B, 2017, 121 (49), pp.11189-11197. 10.1021/acs.jpcb.7b09989 . hal-01682245

\section{HAL Id: hal-01682245 \\ https://hal.sorbonne-universite.fr/hal-01682245}

Submitted on 12 Jan 2018

HAL is a multi-disciplinary open access archive for the deposit and dissemination of scientific research documents, whether they are published or not. The documents may come from teaching and research institutions in France or abroad, or from public or private research centers.
L'archive ouverte pluridisciplinaire HAL, est destinée au dépôt et à la diffusion de documents scientifiques de niveau recherche, publiés ou non, émanant des établissements d'enseignement et de recherche français ou étrangers, des laboratoires publics ou privés. 


\title{
Ab Initio Simulations of Water Dynamics in
} Aqueous TMAO Solutions:

\section{Temperature and Concentration Effects}

\author{
Guillaume Stirnemann, ${ }^{*, \dagger}$ Elise Duboué-Dijon, ${ }^{\ddagger}$ and Damien Laage*,+, \\ †CNRS Laboratoire de Biochimie Théorique, Institut de Biologie Physico-Chimique, Univ. \\ Paris Diderot, Sorbonne Paris Cité, PSL Research University, 13 rue Pierre et Marie \\ Curie, 75005 Paris, France \\ ‡PASTEUR, Département de Chimie, École Normale Supérieure, UPMC Univ Paris 06, \\ CNRS, PSL Research University, 75005 Paris, France \\ \Sorbonne Universités, UPMC Univ Paris 06, ENS, CNRS, PASTEUR, 75005 Paris, \\ France
}

E-mail: stirnemann@ibpc.fr; damien.laage@ens.fr 


\begin{abstract}
We use ab initio molecular dynamics simulation to study the effect of hydrophobic groups on the dynamics of water molecules in aqueous solutions of trimethylamine $\mathrm{N}$-oxide. We show that hydrophobic groups induce a moderate $(<2$-fold $)$ slowdown of water reorientation and hydrogen-bond dynamics in dilute solutions, but that this slowdown rapidly increases with solute concentration. In addition, the slowdown factor is found to vary very little with temperature, thus suggesting an entropic origin. All these results are in quantitative agreement with prior classical molecular dynamics simulations and with the previously suggested excluded-volume model. The hydrophilic TMAO headgroup is found to affect water dynamics more strongly than the hydrophobic moiety, and the magnitude of this slowdown is very sensitive to the strength of the water-solute hydrogen-bond.
\end{abstract}

\title{
Introduction
}

The behavior of water molecules in contact with hydrophobic groups is essential for a broad range of biochemical processes, including for example protein-ligand binding and the assembly of phospholipid membranes. While water molecules were long believed to form iceberglike cages around hydrophobic groups, ${ }^{1}$ there is now a growing body of evidence showing that this picture is exaggerated and not adequate. ${ }^{2-5}$ However, no consensus has yet been reached on the extent of the slowdown induced by hydrophobic groups on the dynamics of vicinal water molecules, which have thus been studied with a broad range of experimental $^{4,6-10}$ and theoretical ${ }^{5,11-14}$ approaches. While some studies suggest that the retardation factor is modest, ${ }^{4,5}$ typically less than 2 , other studies conclude that water molecules are dramatically, e.g. 50-fold, ${ }^{9}$ slowed, or even immobilized. ${ }^{15}$

The conclusions of molecular dynamics simulations are contrasted. While several molecular dynamics (MD) simulations suggested ${ }^{5,11,16}$ that the slowdown of water dynamics is moderate, some subsequent ab initio molecular dynamics (aiMD) simulations ${ }^{12,17}$ found some 
very slow water molecules in aqueous solutions of amphiphilic solutes, and questioned the ability of classical MD to correctly describe the hydration dynamics around hydrophobic groups.

Here we present a systematic aiMD study of hydrophobic hydration dynamics. The paradigm amphiphilic solute that we consider is trimethylamine $N$-oxide (TMAO), whose hydrophobic moiety includes three methyl groups, and which has already been extensively studied experimentally ${ }^{4,6-9}$ and via simulations. ${ }^{5,14}$ TMAO is an osmolyte used by organisms to protect the structure of proteins. A very large body of simulation work, of which we can only give a very brief account, has thus been devoted to determining its hydration shell properties (see e.g. refs. 18,19 and ref. 20 for a recent review), designing adequate forcefields ${ }^{21-26}$ and investigating its interactions with model polymers ${ }^{25,27-30}$ and proteins. ${ }^{26,31-33}$

We will also examine the validity of the excluded volume model that has been suggested, ${ }^{5}$ based on classical MD, to explain and quantify the origin of the dynamical retardation. This model has stressed the great sensitivity of the slowdown in water dynamics vis-a-vis the solute concentration, and its very weak temperature dependence due to its entropic nature. We will therefore use aiMD simulations to determine the concentration and temperature dependence of the effect of TMAO on the hydration dynamics next to the solute's hydrophobic and hydrophilic groups. We will finally discuss and contrast the aiMD results with prior classical MD results.

The outline of the remainder of this paper is as follows: we first detail our aiMD simulation methodology and how water dynamics are analyzed. We then successively examine the dynamics of water in the bulk, next to the TMAO hydrophobic methyl groups at two different temperatures and in dilute and concentrated solutions. We finally analyze the effect of TMAO's hydrophilic head on the water dynamics, before offering some concluding remarks. 


\section{Methods}

\section{Simulations}

$\mathrm{Ab}$ initio molecular dynamics (aiMD) simulations of several solutions of TMAO in $\mathrm{H}_{2} \mathrm{O}$ were performed with the CP2K code, ${ }^{34}$ using the Quickstep implementation ${ }^{35}$ of a hybrid Gaussian plane-wave scheme. ${ }^{36}$ Our methodology followed the recommendations of a previous aiMD study of water dynamical properties. ${ }^{37}$ Energies and forces were described by the BLYP density functional, ${ }^{38,39}$ with Grimme's D3 corrections for dispersion interactions. ${ }^{40}$ We employed a triple-zeta doubly polarized basis set (TZV2P), a 400 Ry cutoff for the plane-wave grid, and the Goedecker-Teter-Hutter norm-conserving pseudopotentials. ${ }^{41}$ BornOppenheimer molecular dynamics simulations were performed in the canonical ensemble: the temperature was controlled using a Nose-Hoover thermostat, and the density was fixed at its

extrapolated experimental value. ${ }^{33}$ Systems were first equilibrated via classical MD simulations using a classical TMAO forcefield ${ }^{21}$ and the SPC/E water model. ${ }^{42}$ The dilute solution contained 1 TMAO and 124 water molecules, with a box size of $15.6556 \AA$ at $300 \mathrm{~K}$ and $15.7040 \AA$ at $323 \mathrm{~K}$. The concentrated solution was made of 12 TMAO molecules solvated by 83 water molecules, in a cubic box of $15.7376 \AA$-side. These systems respectively correspond to $0.4 \mathrm{~mol} / \mathrm{L}$ and $5.1 \mathrm{~mol} / \mathrm{L}$ concentrations, and $0.4 \mathrm{~mol} / \mathrm{kg}$ and $8.0 \mathrm{~mol} / \mathrm{kg}$ molalities. Simulations were performed for 80 ps for the dilute solution at $323 \mathrm{~K}$ and the concentrated solution at $300 \mathrm{~K}$, whereas the dilute solution at $300 \mathrm{~K}$ was simulated for $160 \mathrm{ps}$. In each case, the first 10 ps of the simulations were discarded from the subsequent analysis.

\section{Analysis}

\section{Shell definitions}

Geometric criteria were employed to define the different parts of the TMAO hydration shell (see Fig. 1A). The hydrophobic part of the TMAO hydration shell (OH groups with blue hydrogen atoms in Fig. 1A) includes all water $\mathrm{OH}$ groups whose oxygen atom is within 
$4.4 \AA$ of any of the three TMAO carbon atoms and whose hydrogen atom is farther than $2.1 \AA$ from the TMAO oxygen atom. The hydrophilic part of the hydration shell (OH groups with red hydrogen atoms in Fig. 1A) includes all water $\mathrm{OH}$ groups donating a hydrogen bond to the TMAO oxygen headgroup; it is defined by a maximum distance of $2.1 \AA$ between the water hydrogen atom and the TMAO oxygen atom, corresponding to the first minimum in the radial distribution function between these atoms (due to the great strength of this H-bond, additional geometric criteria are not necessary here). Finally, the bulk-like region includes $\mathrm{OH}$ groups whose oxygen atom is farther than $6 \AA$ from any TMAO central nitrogen atom.

\section{Reorientation and H-bond jump dynamics}

The water reorientation dynamics are probed by the reorientation time-correlation function (tcf)

$$
C_{\text {reor }}(t)=\left\langle P_{2}\left[\mathbf{u}_{\mathrm{OH}}(0) \mathbf{u}_{\mathrm{OH}}(t)\right]\right\rangle,
$$

where $\mathbf{u}_{\mathrm{OH}}(t)$ is the orientation of the water $\mathrm{OH}$ group at time $t$ and $P_{2}$ is the second order Legendre polynomial. The reorientation time $\tau_{\text {reor }}$ is determined from an exponential fit of $C_{\text {reor }}(t)$ to $A e^{-t / \tau_{\text {reor }}}$ for $1 \leq t \leq 15$ ps. Uncertainties on $C_{\text {reor }}(t)$ were determined by calculating $C_{\text {reor }}(t)$ on 4 independent blocks ( 5 for the $300 \mathrm{~K}$ dilute simulation) and determining the standard deviation. The shell and bulk-like reorientation tcfs were determined by considering the location of the water $\mathrm{OH}$ group at time $t=0$. To estimate the putative plateau of the tcfs at very long times if 2 water molecules per methyl group were immobilized, we considered the total fraction of such molecules ( 6 out of $\approx 14$ in the hydrophobic shell ${ }^{21}$ ) that would not reorient on long timescales, but still experience a fast librational decay (which accounts for $\approx 20-25 \%$ of the total decay $\left.{ }^{43}\right)$.

The jump dynamics of $\mathrm{OH}$ groups between $\mathrm{H}$-bond acceptors are probed by the jump time-correlation function ${ }^{44}$

$$
C_{\text {jump }}(t)=1-\left\langle p_{I}(0) p_{F}(t)\right\rangle
$$


where $p_{I}(t)$ is 1 if the $\mathrm{OH}$ group is stably $\mathrm{H}$-bonded to the initial acceptor $I$ at time $t$ and 0 otherwise, and correspondingly for the probability $p_{F}(t)$ to be stably H-bonded with the final acceptor. This jump tcf can be understood as a H-bond survival probability since it probes the kinetics to switch between stable H-bond acceptors. Stable H-bonds are defined within the Stable States Picture to remove contributions from fast barrier recrossing, and absorbing boundary conditions in the final state $F$ are applied. ${ }^{44}$ Stable H-bonds were defined using the following geometric criteria: ${ }^{5}$ the distance between the donor and acceptor oxygen atoms should be less than $3.0 \AA$, that between the donor hydrogen and the acceptor oxygen atoms smaller than $2.0 \AA$, and the angle between $\mathrm{OH}$ and the $\mathrm{OO}$ axis less than $18^{\circ}$. The jump time $\tau_{j}$ is determined from an exponential fit of $C_{\text {jump }}(t)$ for $1 \leq t \leq 20 \mathrm{ps}$. We have checked that alternative fitting procedures, e.g. with a multi exponential function, lead to very similar jump time values.

\section{Results and discussions}

\section{Bulk water dynamics}

Previous aiMD studies ${ }^{45-48}$ have shown that many DFT functionals, including the BLYP functional used here, tend to over-structure liquid water under ambient conditions. This has a dramatic effect on the simulated dynamical properties, including e.g. water diffusion which was found to be much slower than measured experimentally. ${ }^{45-48}$ Semi-empirical corrections aiming at a better description of dispersion interactions ${ }^{40}$ have been applied in order to solve this issue. However, for many water properties, these corrections do not totally suppress the discrepancies between the results obtained from aiMD simulations and experiments performed at the same temperature. ${ }^{37}$ With the popular BLYP-D3 simulation scheme employed here, it has thus become common practice to increase the simulation temperature by $\approx 20 \mathrm{~K}$ to reproduce experimental observables, ${ }^{13,14,37}$ including e.g. the radial distribution functions and the translational diffusion coefficient. ${ }^{37}$ However, the magnitude 
of this temperature increase was determined for neat liquid water. In contrast, in the TMAO aqueous solutions studied here, different H-bonds are present, with different strengths, different activation energies, and thus different responses to a temperature change. This has motivated our choice to repeat our study of water dynamics in aqueous TMAO solutions at two different temperatures, $300 \mathrm{~K}$ and $323 \mathrm{~K}$.

We first compare the reorientation dynamics for bulk-like water molecules calculated from aiMD trajectories at these two different temperatures (see Fig. 1B-C). As expected, at $300 \mathrm{~K}$ the reorientation dynamics are much slower in the aiMD simulation than measured experimentally. The computed bulk reorientation time is $\tau_{2}^{b u l k}=6.2 \mathrm{ps}$, more than twice slower than the experimental value $\left(\tau_{2}^{\text {bulk }} \approx 2.5 \mathrm{ps}\right)$ obtained by ultrafast infrared spectroscopy. ${ }^{49}$ A recent study reported $\mathrm{a} \approx 40 \%$-slowdown of the water translational diffusion coefficient at $300 \mathrm{~K}$ when comparing experiments and classical MD simulations based on neural network potentials calibrated to reproduce BLYP-D3 simulations. ${ }^{50}$ In contrast, when the temperature is increased to $323 \mathrm{~K}$ (Fig 1C), the bulk-like reorientation time value decreases to reach $\tau_{2}^{\text {bulk }}=2.6 \mathrm{ps}$, in good agreement with the $2.5 \mathrm{ps}$ experimental value. ${ }^{49} \mathrm{~A}$ similar agreement was already observed in prior aiMD simulations for the reorientation dynamics in heavy ${ }^{14}$ and normal ${ }^{13}$ water, and the translational dynamics in heavy water. ${ }^{37}$

In the following, we will examine the TMAO hydration shell reorientation dynamics at these two temperatures, considering both the hydrophobic and hydrophilic regions of the hydration shell. We will focus on the effect induced by the solute on the reorientation dynamics of the surrounding water molecules. We will therefore mainly consider the ratio between the reorientation times in the shell and in the bulk, more than the absolute values of these times, which, as shown above, are not quantitatively described by aiMD.

\section{Hydrophobic hydration shell dynamics in a dilute solution}

We first study the effect of the TMAO hydrophobic moiety on the hydration shell reorientation dynamics. The reorientation tcf eq 1 at $300 \mathrm{~K}$ for all water $\mathrm{OH}$ groups next to the three 

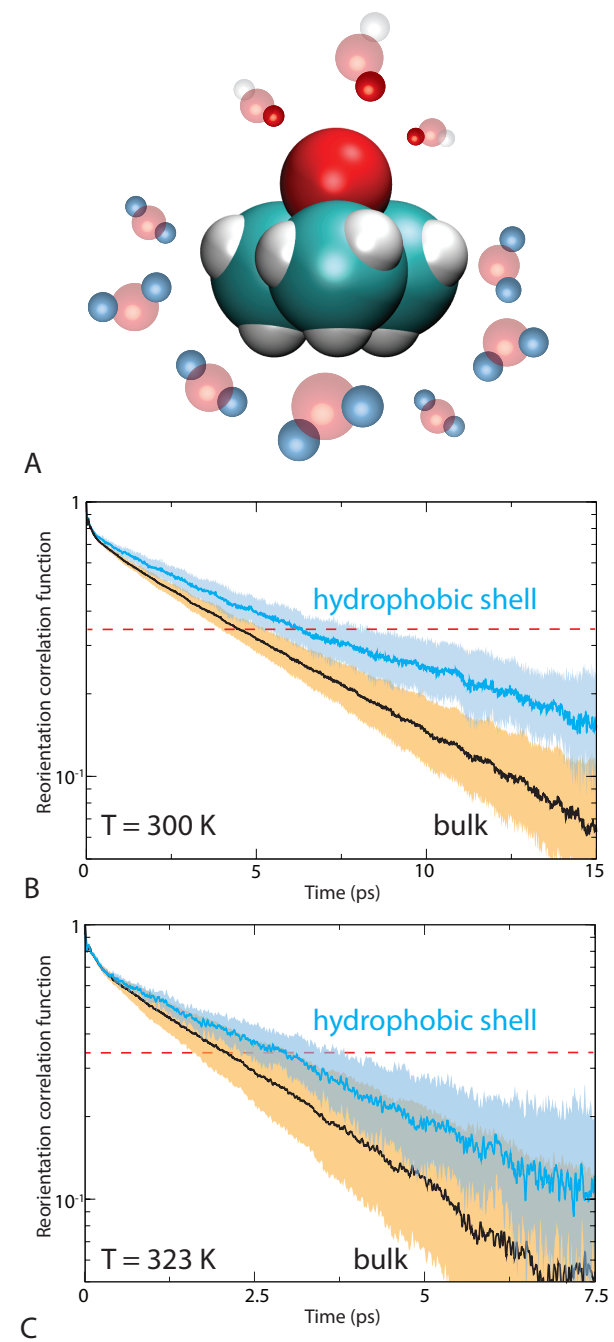

Figure 1: Definition of $\mathrm{OH}$ populations and reorientation dynamics in dilute hydrophobic shells (A) Scheme of a TMAO molecule with the hydration shell regions solvating its hydrophobic ( $\mathrm{OH}$ groups with blue hydrogen atoms) and hydrophilic moieties $(\mathrm{OH}$ groups with red hydrogen atoms). (B) Reorientation time-correlation functions eq 1 in the dilute aqueous TMAO solution at $300 \mathrm{~K}$ for water molecules initially lying in the hydrophobic hydration shell (blue) and in the bulk-like region (black). The solid lines are the average decays and the shaded orange and blue regions show the amplitude of the fluctuations obtained by block-averaging. The fast initial decay is due to librations. ${ }^{43}$ The dashed red lines correspond to the putative plateau of the tcfs at very long times if 2 water molecules per methyl group were immobilized, as suggested in ref 15. (C) idem at $323 \mathrm{~K}$.

hydrophobic TMAO methyl groups is shown in Fig. 1B and is found to decay more slowly than in the bulk. The hydrophobic shell reorientation time is $\tau_{2}^{\text {hydrophobic }}=10.2 \mathrm{ps}$, leading to a slowdown factor of $10.2 / 6.2 \approx 1.6$ relative to the bulk. We first note that similar slowdown values have been obtained in prior aiMD simulations of related systems, including TMAO 
in heavy water, ${ }^{14}$ aqueous solutions of tetramethylurea, ${ }^{13}$ methane ${ }^{51}$ and methanol, ${ }^{16}$ and also experimentally on a series of amphiphilic molecules in aqueous solution. ${ }^{4}$ This therefore suggests that this moderate slowdown of water reorientation dynamics is a common feature of dilute hydrophobic groups; this is consistent with the excluded-volume picture ${ }^{5}$ of hydrophobic hydration dynamics and we will return to this point below. It is also important to stress the quantitative agreement between the slowdown determined from aiMD simulations and that obtained in earlier classical MD simulations on the same system. ${ }^{5,52,53}$ This shows that in contrast to some suggestions, ${ }^{6,12,13,54}$ classical force-fields do provide a good description of this slowdown, which does not require an explicit account of electronic effects (we note that a fair comparison between these approaches involves the reorientation time slowdown factor; in contrast, using the raw reorientation tcf as done in ref 13 is misleading since it is sensitive to the initial librational decay and does not correct for the slow bulk aiMD dynamics).

We now consider the same ensemble of water OH groups solvating the TMAO hydrophobic part, but at the elevated $323 \mathrm{~K}$ temperature. Their reorientation time determined from the reorientation tcf shown in Fig. 1C is $\tau_{2}^{\text {hydrophobic }}=3.9 \mathrm{ps}$, leading to a slowdown factor of $3.9 / 2.6 \approx 1.5$ relative to the bulk. This suggests that the temperature shift used in the aiMD simulations to reproduce the ambient bulk water dynamics does not affect the ratio between the reorientation times in the hydrophobic hydration shell and in the bulk. This can be explained by two factors. First, both bulk-like and hydrophobic shell water $\mathrm{OH}$ groups donate a H-bond to a water oxygen atom and their strengths are very similar, so that the temperature dependence of their dynamics is also similar. Second, it has been shown both by NMR experiments ${ }^{4}$ and by classical MD simulations ${ }^{53}$ that the reorientation slowdown factor between the hydrophobic hydration shell and the bulk is very little temperature-dependent around $300 \mathrm{~K}$. However, as we will show below, the situation is very different for the hydrophilic part of TMAO.

In addition to the magnitude of the slowdown induced by a hydrophobic group, another 
key and much debated question is the number of molecules that are affected. Ultrafast spectroscopy experiments ${ }^{15,55}$ have suggested that only a minor fraction of the hydration shell water molecules are strongly slowed down or even immobilized (on average, 3 or $4 \mathrm{OH}$ groups per methyl group, i.e. typically between one third and one half of the first hydration shell surrounding the hydrophobic groups), while the rest of the shell exhibits bulk-like dynamics. In contrast, classical MD simulations had concluded ${ }^{5}$ that all the hydration shell molecules are affected, but moderately slowed. Our present aiMD results clearly contradict the interpretation of the ultrafast spectroscopy results and support a picture where in dilute conditions the entire shell exhibits a moderate slowdown, as found in classical MD. The reorientation tcfs in Fig 1B-C are averaged over all water $\mathrm{OH}$ groups initially present in the hydrophobic hydration shell. Their quasi-monoexponential decay beyond the initial fast librational reorientation suggests that they all reorient on a similar timescale and that there are no very distinct - bulk-like and strongly retarded - populations.

Further, the average reorientation tcfs in the shell decay well below the value that would correspond to an immobilized fraction of 3-4 water OHs per methyl group, as indicated by a red dashed line in Fig 1B-C. This therefore rules out the presence of a very slow fraction of water molecules. We stress that unambiguous conclusions on this aspect could be reached because the tcfs were calculated up to long delays, with carefully determined uncertainties. This explains the discrepancy with prior ultrafast spectroscopy ${ }^{15,55}$ and aiMD ${ }^{12,17}$ studies which suggested the presence of some very slow water molecules. These latter studies only determined the tcf up to shorter delays (in particular because of the short vibrational lifetime in the experiments). A multiexponential fit was applied, where water dynamics was arbitrarily decomposed at any concentration into a fixed fast timescale, assigned to a bulk-like population, and a fixed slow timescale, assigned to water OHs solvating hydrophobic groups. Such an approach cannot discriminate between many moderately retarded water molecules and a few strongly retarded molecules because the long-time decay is not accessible.

We now turn to the molecular origin of the retarded water dynamics in the hydration 
shell of hydrophobic groups. The extended jump model ${ }^{56}$ combined with an excluded volume approach $^{5}$ can provide an excellent description of this slowdown. Water long-time reorientation was shown to proceed via two mechanisms: ${ }^{56}$ the dominant mechanism involves large angular jumps occuring during H-bond exchanges; an additional minor contribution is due to reorientational diffusion of intact H-bonded pairs between successive jumps. For water molecules in the vicinity of hydrophobic groups, a prior study by two of us based on classical MD simulations,${ }^{5}$ suggested that the water reorientation slowdown is mainly due to a slower rate constant for H-bond jumps between water H-bond acceptors, which stems from an entropic excluded volume effect. ${ }^{5}$ The solute(s) hinder(s) the approach of a new water partner that can accept an H-bond from the reorienting $\mathrm{OH}$. A small convex solute usually occupies $\approx 1 / 3$ of the local space available for the new partner, leading to a slowdown of $\approx 1.5$, while a flat extended hydrophobic surface blocks half of the space, and thus leads to a 2 -fold slowdown of water reorientation. ${ }^{57} \mathrm{~A}$ transition state excluded volume (TSEV) model $^{5}$ was developed to quantitatively describe and explain the slower water dynamics in many different environments, including dilute and concentrated solutions of amphiphilic molecules, ${ }^{5,52,58}$ extended hydrophobic surfaces ${ }^{57}$ and confining pores. ${ }^{59,60}$ We note that the frame component, i.e. the reorientation between the jumps, also slows down in the presence of hydrophobic groups, ${ }^{5}$ but it remains a minor contribution to the reorientation time. As will be shown below, in the presence of H-bond acceptor groups, e.g. in aqueous solutions of amphiphilic molecules, ions ${ }^{61,62}$ and proteins, ${ }^{63}$ an additional factor accounting for the H-bond strength affects the jump time. ${ }^{64}$

We therefore now focus on the water H-bond jump times in the dilute TMAO solutions. They are determined from the survival probabilities of stable H-bonds, shown in Fig 2B-C. Prior classical MD simulations of neat liquid water with force-fields which are established to provide a good description of ambient water dynamics found jump time values of $3.3 \mathrm{ps}$ for the SPC/E model ${ }^{5,44}$ and 4.4 ps for the TIP4P/2005 model. ${ }^{65}$ Our aiMD simulations yield H-bond jump times of $6.6 \mathrm{ps}$ and $4.1 \mathrm{ps}$ for bulk water at $300 \mathrm{~K}$ and $323 \mathrm{~K}$, respectively. 

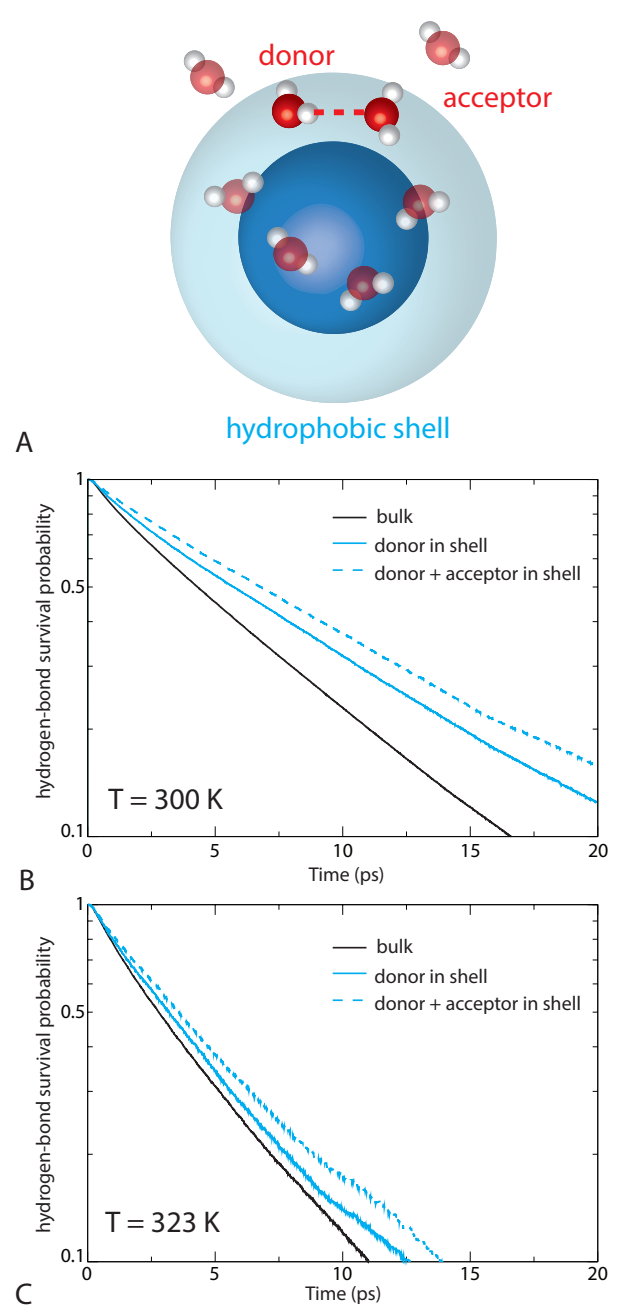

Figure 2: An excluded volume effect explains the slower H-bond exchange probability in TMAO's hydrophobic hydration shell (A) Schematic representation of the excluded volume induced by a hydrophobic solute (dark blue) on water H-bonds present in the first hydration shell (light blue). (B) Water jump H-bond exchange tcf eq 2 in different environments for a dilute TMAO aqueous solution at $300 \mathrm{~K}$ : both the water $\mathrm{OH}$ group and its $\mathrm{H}$-bond acceptor are in the bulk (black line), water $\mathrm{OH}$ group in the hydration shell of TMAO's hydrophobic groups (plain blue line), and both the $\mathrm{OH}$ group and its H-bond acceptor in the hydrophobic hydration shell (dashed blue line). (C) Same data at 323 K.

As for the reorientation time, the aiMD simulations yield dynamics that are too slow at $300 \mathrm{~K}$, but the temperature shift allows to recover the ambient dynamics. We now turn to the H-bond jump times in the hydration shells of the TMAO hydrophobic groups. At both temperatures, the H-bond jump dynamics exhibit a moderate retardation in the shell relatively to the bulk (Fig $2 \mathrm{~B}-\mathrm{C}$ ), with slowdown factors of $\approx 1.3$ at $300 \mathrm{~K}$ and $\approx 1.1$ at 
$323 \mathrm{~K}$. This effect is more pronounced when one focuses on the jump dynamics of OH groups which are in the shell and which donate a H-bond to another water molecule in the shell, i.e. when the $\mathrm{OH}$ group is tangent to the hydrophobic group $(1.5$ at $300 \mathrm{~K}$ and 1.3 at at $323 \mathrm{~K})$. These results are quantitatively explained by TSEV considerations. Tangent OHs experience a larger excluded volume factor and for a solute with the size of TMAO they are expected to be retarded by a factor 1.5 , as already confirmed by classical MD simulations. ${ }^{5}$ The average over the entire hydration shell includes some tangent $\mathrm{OHs}$, and some OHs pointing away from the solute, towards an H-bond acceptor in the bulk and which are thus very moderately affected because the TSEV factor is close to $1 .{ }^{5}$ As a consequence, when averaged over the entire hydrophobic hydration shell, the observed slowdown of the jump time is moderate and close to 1.3 (Figure 2).

While the slight decrease in the jump and reorientation retardation factors that is observed as the temperature increases from $300 \mathrm{~K}$ to $323 \mathrm{~K}$ is in agreement with experimental ${ }^{4}$ and classical simulation ${ }^{53}$ results, its very small amplitude is close to the uncertainties on these values. While the excluded volume effect is solely entropic and therefore predicts a temperature-independent slowdown, the model can be further complemented to account for the subtle changes in the local tetrahedral order around the bulk and shell water molecules, which was shown to quantitatively describe this very weak temperature dependence at ambient conditions. ${ }^{53}$

We note that several recent classical ${ }^{66}$ and ab initio ${ }^{13}$ molecular dynamics studies of water dynamics next to hydrophobic groups have obtained results which are consistent with our jump model picture with excluded volume. The main difference with our present approach is that they consider the fate of transiently broken H-bonds to identify which ones return to the original H-bond acceptor (a failed jump attempt in our description) and which ones switch to a different acceptor (a successful jump). The main limitation of this approach is that it relies on very arbitrary H-bond definitions, while our stable states picture solves this issue by directly considering the successful jumps between stable H-bonds and is largely 
independent of the H-bond definition. ${ }^{44}$ However, in all these descriptions, reorientation is described according to the original jump model as arising from large amplitude jumps and from a slower reorientation of intact H-bonds between successive jumps. ${ }^{44}$

As an intermediate conclusion, our aiMD results therefore suggest that in dilute aqueous solutions, water reorientation is moderately slowed in the hydration shell of the TMAO hydrophobic methyl groups. The moderate $\approx 1.5$ retardation factor is mainly due to a slowdown in the H-bond jump rate constant, arising from an entropic excluded volume effect. These results are in quantitative agreement with our earlier classical MD simulations. ${ }^{5,52}$ A similar slowdown is expected with the broad range of classical forcefields ${ }^{21-26}$ that have been recently suggested for TMAO, because it is determined by the volume excluded by the methyl groups, and the latter have similar van der Waals radii in the different forcefields. While the DFT functional leads to an overstructured liquid and thus to overestimated reorientation times, the retardation factors are identical to those obtained by classical MD simulations. There is thus no sign of peculiar dynamical features that would be absent or not well reproduced by classical forcefields. In particular, we do not find any evidence of distinct $\mathrm{OH}$ populations ${ }^{15}$ in the hydrophobic hydration shell that would reorient at very different rates. ${ }^{12,17}$

\section{Hydrophobic hydration shell dynamics in concentrated solutions}

We now investigate how the solute concentration affects the water reorientation dynamics in the hydrophobic hydration shell. We considered a concentrated $8 \mathrm{~m}$ TMAO solution, which is a typical concentration used in ultrafast spectroscopy experiments. ${ }^{15}$ The reorientation tcf (eq 1) calculated from our aiMD simulation at $300 \mathrm{~K}$ for all water $\mathrm{OH}$ groups lying next to the TMAO hydrophobic groups is shown in Fig 3A. It reveals two important points. First, water reorientation in the shell is significantly slower in concentrated conditions than in the dilute solution (we stress that this is not averaged over the entire solution but determined specifically for the TMAO hydrophobic hydration shell): the slowdown relative to the bulk is $\approx 5.8$, which contrasts with the moderate $\approx 1.6$ slowdown in the dilute $300 \mathrm{~K}$ solution. 
Second, the tcf long-time decay exhibits a pronounced non-exponential behavior, while it was quasi-exponential in the dilute case, thus suggesting the presence of a broad distribution of slowdown factors. These results confirm that the effect of hydrophobic groups on water dynamics is strongly concentration dependent, as already observed in classical MD simulations ${ }^{5,52,58}$ and as quantitatively predicted by the TSEV model. ${ }^{5}$ As the concentration in hydrophobic groups increases, the excluded volume occupied by the solute molecules grows (see Fig 3B where each $\mathrm{OH}$ is surrounded by several solutes ${ }^{58}$ ); this increasingly large excluded volume hinders the approach of new H-bond partners and slows down the jump rate constant. The analysis of previous classical simulations performed at the same concentration showed that the slowdown factor expected from the TSEV model is $\approx 4-5,{ }^{58}$ which is in good agreement with the 5.8 value found in the present aiMD simulation. In addition, there is a much greater variety of local environments experienced by water $\mathrm{OH}$ groups in concentrated solutions than in the dilute case. In dilute solutions, a water $\mathrm{OH}$ group within the TMAO methyl group hydration shell is affected by a single solute at a time and is in a well-defined geometry, tangent to the hydrophobic group and surrounded by bulk water. In contrast, in concentrated solutions, a given water $\mathrm{OH}$ group can be in the vicinity of one or several solutes simultaneously. Classical MD simulations have shown that the variety of local structures gave rise to a broad distribution of excluded volumes. ${ }^{58}$ This explains the increasingly non-exponential character of the $\mathrm{OH}$ reorientation tcf, as also observed in our aiMD results (Fig 3A). In addition, by connecting the local solution structure and water reorientation dynamics, the TSEV model can also explain how the retardation induced by amphiphilic solutes at increasing concentrations crucially depends on the molecules tendency to aggregate. TMAO molecules do not aggregate up to very high concentrations, so that their hydration shells strongly overlap and the water reorientation dynamics are significantly retarded. In contrast, amphiphilic solutes such as tetramethylurea (TMU) form clusters at high concentrations ${ }^{58}$ that surround more dilute regions. As a consequence, the observed effect on water reorientation dynamics is less pronounced than for TMAO. ${ }^{58}$ 

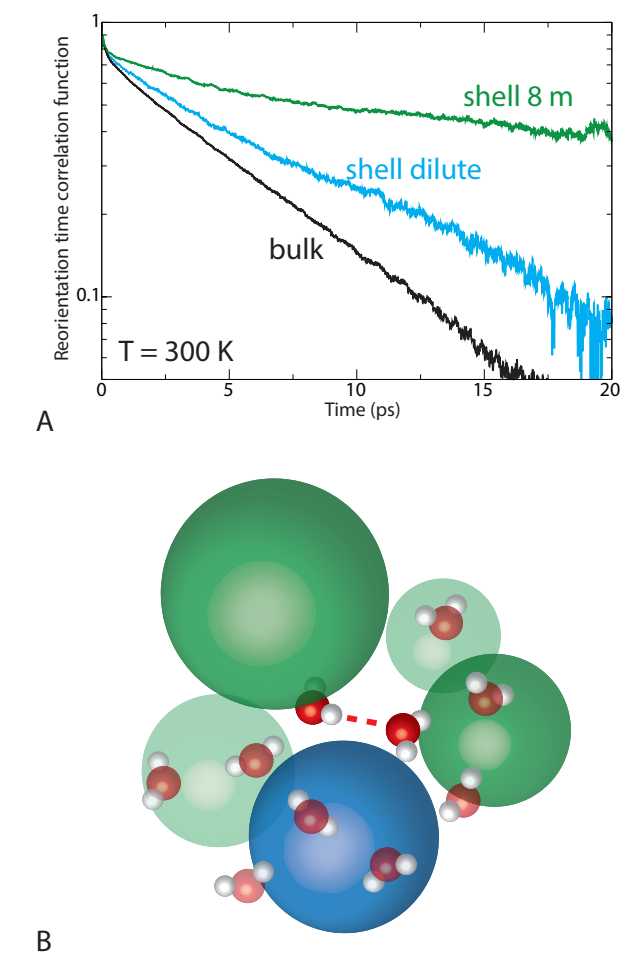

Figure 3: The excluded volume effect explains the large retardation at high concentration (A) Reorientation tcf Eq 1 of the water $\mathrm{OH}$ groups at $300 \mathrm{~K}$ in the bulk (black), in the hydrophobic hydration shell of a dilute TMAO molecule (blue) and of a concentrated, $8 \mathrm{~m}$ TMAO solution (green). (B) Schematic representation of the excluded volume effect caused by hydrophobic groups in a dilute (blue) and concentrated (blue and green) solution.

\section{Hydrophilic hydration shell dynamics}

While the hydration dynamics of the three TMAO methyl groups have been extensively studied as a paradigm hydrophobic hydration case, there is now a growing interest for TMAO's hydrophilic headgroup and its effect on water dynamics, due to its putative connection to TMAO's protein structure protecting role. ${ }^{20}$ TMAO is amphiphilic, and contains a hydrophilic oxygen headgroup which bears a pronounced negative partial charge and can accept two to three H-bonds from water molecules. The reorientation tcfs eq 1 computed from our aiMD simulations for water $\mathrm{OH}$ groups initially $\mathrm{H}$-bonded to the TMAO oxygen site in a dilute solution are found to be strongly slowed down compared to those of bulk-like $\mathrm{OH}$ groups, both at $300 \mathrm{~K}$ and at $323 \mathrm{~K}$ (Figure 4A). The respective retardation factors are 5.2 
and 4.1.

This slowdown next to a hydrophilic site differs in a several key aspects from the slowdown next to hydrophobic groups that we discussed above. First, its magnitude is much greater (at $300 \mathrm{~K}, \approx 5.2$ vs $\approx 1.6$ for the dilute hydrophobic slowdown). Second, its value is very sensitive to the details of the description: while the $>4$ value found here is similar to prior aiMD determinations at $320 \mathrm{~K}$ using three different DFT functionals in heavy water, ${ }^{14}$ it exceeds the values found in prior $300 \mathrm{~K}$ classical MD simulations, which ranged from $2.5^{5,52}$ for the forcefield of ref 22 to $1.5-1.8^{14,67}$ for that of ref 21 (more recent TMAO classical forcefield reparameterizations lead to values in the same $2-3^{67}$ range). In contrast, the hydrophobic slowdown factor in dilute conditions is consistently found to be approximately 1.5 in both classical and aiMD simulations. Third, the hydrophilic slowdown exhibits a pronounced temperature dependence (5.2 at $300 \mathrm{~K}$ vs. 4.1 at $323 \mathrm{~K}$ ), while the hydrophobic slowdown factor is almost unchanged between these two temperatures $(1.6$ at $300 \mathrm{~K}$ vs. 1.5 at $323 \mathrm{~K})$.

These three key differences between hydrophobic and hydrophilic slowdown factors can be understood by the effects of these different chemical groups on the water H-bond jumps. ${ }^{43}$ A molecular picture of these effects was provided by the extended jump mechanism, complemented by the excluded-volume effect induced by all solutes (both hydrophobic and hydrophilic) and by an H-bond strength effect specific to solutes which are H-bond acceptors. ${ }^{43}$

This model shows that for water molecules that are initially donating an H-bond to a solute site, their H-bond jump time is not only slowed by the solute-induced excludedvolume (TSEV) effect - as described above for hydrophobic groups - but it is also affected by the strength of this $\mathrm{H}$-bond with the solute that needs to be elongated to reach the jump transition state. Solutes that accept weaker H-bonds than water-water bonds facilitate the jumps while strong H-bond acceptors slow down the jumps. ${ }^{64}$ The net effect on the water jump dynamics is a combination of the excluded-volume slowdown and of the H-bond strength effect. As shown in prior studies on ions, ${ }^{62}$ amino-acids ${ }^{64}$ and proteins, ${ }^{63}$ some sites 

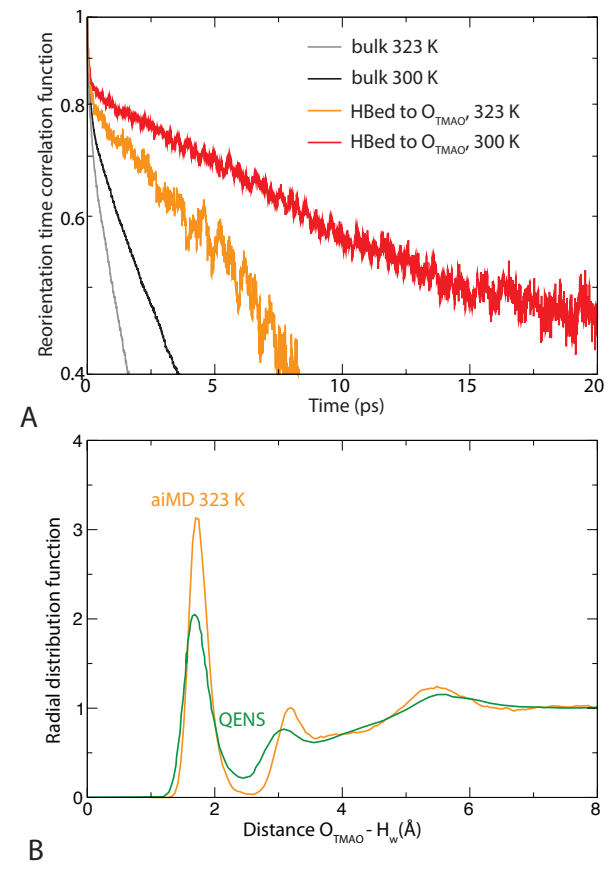

Figure 4: Structure and dynamics in the hydrophilic shell (A) Reorientation tcf eq 1 of the bulk-like water $\mathrm{OH}$ groups at $300 \mathrm{~K}$ (black) and $323 \mathrm{~K}$ (gray), and for the water $\mathrm{OH}$ groups initially $\mathrm{H}$-bonded to a TMAO oxygen atom at $300 \mathrm{~K}$ (red) and $323 \mathrm{~K}$ (orange) in a dilute solution. (B) Radial distribution functions between the oxygen of TMAO and hydrogen atoms of water molecules, determined from $323 \mathrm{~K}$ aiMD simulations (orange) and from neutron scattering experiments ${ }^{68}$ (green).

can accelerate the jump dynamics while others slow them down.

For TMAO, the large $>4$ slowdown factor found here next to the TMAO oxygen site which largely exceeds the typical $<2$ excluded volume slowdown in dilute solutions- thus arises from a very strong water-TMAO H-bond. This strong H-bond also manifests itself in the $\mathrm{O}_{\mathrm{TMAO}}-\mathrm{H}_{w}$ radial distribution function (Fig 4B), which shows a very sharp peak at the position of the first hydration shell, and a very deep minimum in the interstitial region between the first and second shells. Consequently, the resulting free energy barrier to break this H-bond is expected to be much larger than that of a water-water H-bond. This effect is present but less pronounced in classical force-field descriptions of TMAO, ${ }^{5,14}$ because this slowdown is very sensitive to the strength of the water-TMAO interaction for each set of parameters. This contrasts with hydrophobic groups that only interact with water through their excluded volume, which is consistently described by classical and aiMD simulations. A 
further consequence of this strong water-TMAO H-bond is that its jump activation energy is larger than the bulk water jump activation energy. This therefore explains the marked temperature dependence of the slowdown factor.

Both our present aiMD results and prior classical ${ }^{52}$ and aiMD ${ }^{14}$ simulations suggest that the hydrophilic head of TMAO affects water reorientation and H-bond dynamics more strongly than the hydrophobic groups. This is in agreement with recent interpretations of fsIR and $\mathrm{THz}$ measurements, ${ }^{6,20}$ and shows that the conclusions of earlier fsIR experiments ${ }^{15,55,69,70}$ which had assumed that the hydrophilic heads of TMAO and other amphiphiles had no significant effect on water dynamics should be revised. As shown in ref 52 this strong TMAO-water H-bond also explains the large red-shift of the water $\mathrm{OH}$ vibrational frequency observed in the IR spectra.

While all recent studies concur to conclude that the TMAO hydrophilic head strongly retards water dynamics, the exact magnitude of this slowdown remains uncertain. Very few experiments have probed the hydrophilic head hydration shell. Dielectric spectroscopy experiments ${ }^{6}$ suggested that TMAO forms a very long-lived complex with two or three water molecules that tumble together with TMAO in the solution. However, these results ${ }^{6}$ relied on the effective dipole moment value obtained with a very simplified Cavell equation and assumed that the solute is spherical and not polarizable. The conclusions may thus change if these severe approximations are not used.

Regarding simulations, the induced slowdown was found to be systematically smaller in classical MD simulations than in aiMD. However, while aiMD provides a more sophisticated description of the interactions than classical MD, it exhibits important discrepancies with the experiments. This is visible in the radial distribution function (rdf) between water hydrogens and TMAO's oxygen atom. Figure 4B shows that the results obtained from the $323 \mathrm{~K}$ aiMD simulation (the temperature at which the bulk dynamics match experimental $298 \mathrm{~K}$ measurements) and from $298 \mathrm{~K}$ quasi-elastic neutron scattering (QENS) experiments ${ }^{68}$ ex- $^{\text {ex }}$ hibit important differences. The jump free energy barrier was shown to be mostly due to 
the free energy cost to elongate the initial H-bond and contract the future H-bond, which both can be determined from such a radial distribution function. ${ }^{71}$ Figure $4 \mathrm{~B}$ shows that the first peak is higher and the first minimum lower in the aiMD than in the QENS results, thus strongly increasing the jump free energy barrier and the lifetime of the TMAO-water H-bond. While the QENS rdf is not measured directly but obtained by an empirical potential structure refinement method that reproduces the experimental structure factors, it strongly suggests that current aiMD descriptions overestimate the TMAO-water H-bond strength. An additional limitation of the aiMD approach for solutes in aqueous solutions is the presence of several types of H-bonds with different activation energies. The widely employed temperature shift correction was calibrated on neat liquid water, and the proper dynamics will not be recovered for water-solute H-bonds whose strength differs from that of water-water bonds, as is the case here for the TMAO-water bonds.

It is therefore not obvious that current aiMD results should be used as a reference to parameterize new classical TMAO force-fields. Recent efforts have however been made in this direction, mostly to increase the strength of the water-TMAO H-bond. It has also been argued that TMAO-water H-bonds in classical force-fields should be more directional, and that an explicit description of lone pairs was needed to orient water molecules around TMAO's hydrophilic head. ${ }^{14}$ However, we note that conventional three-site water models (e.g. SPC/ $\mathrm{E}^{42}$ ) provide a reasonable description of water's angular structure and dynamics, with no need of explicit representation of molecular orbitals, and that an increase in the absolute charge ${ }^{24}$ on TMAO's oxygen was shown to yield results in better agreement with aiMD,${ }^{67}$ without introducing any directionality.

\section{Conclusion}

We have performed aiMD simulations of aqueous solutions of TMAO at different temperatures and concentrations. Our results show that, in perfect agreement with prior classical 
MD simulations, hydrophobic groups in dilute conditions induce a moderate $<2$ slowdown in the reorientation and H-bond dynamics of their hydration-shell water molecules relative to the bulk. This slowdown factor is found to markedly increase in concentrated solutions. Its very weak temperature dependence suggests an entropic origin of this slowdown. All these features are consistent with the excluded volume model, ${ }^{5}$ which identifies the molecular origin of this retardation and quantitatively relates it to the hindrance of H-bond jump exchanges due to the solute presence. Our analysis of water dynamics next to the hydrophilic oxygen headgroup of TMAO shows that in agreement with prior studies, the strong waterTMAO H-bond leads to a pronounced slowdown relative to the bulk. However, the extent of this slowdown is very sensitive to the H-bond interaction potential, and our results suggest that current aiMD simulations may overestimate this H-bond strength.

\section{Acknowledgement}

Rodolphe Vuilleumier and Ari Seitsonen (ENS) are gratefully aknowledged for their suggestions regarding the aiMD simulation setup. This work was performed using HPC resources from GENCI-TGCC (Grant 2014077156). The research leading to these results has received funding from the European Research Council under the European Unions Seventh Framework Program (FP7/2007-2013)/ERC Grant Agreement No. 279977 (D.L.) and this work was supported by the "Initiative d'Excellence" program from the French State (Grant "DYNAMO", ANR-11-LABX-0011-01 to GS).

\section{References}

(1) Frank, H. S.; Evans, M. W. Free Volume and Entropy in Condensed Systems III. Entropy in Binary Liquid Mixtures; Partial Molal Entropy in Dilute Solutions; Structure and Thermodynamics in Aqueous Electrolytes. J. Chem. Phys. 1945, 13, 507-532. 
(2) Blokzijl, W.; Engberts, J. B. F. N. Hydrophobic Effects. Opinions and Facts. Angew. Chem. Int. Edit. Engl. 1993, 32, 1545-1579.

(3) Buchanan, P.; Aldiwan, N.; Soper, A. K.; Creek, J. L.; Koh, C. A. Decreased Structure on Dissolving Methane in Water. Chem. Phys. Lett. 2005, 415, 89-93.

(4) Qvist, J.; Halle, B. Thermal Signature of Hydrophobic Hydration Dynamics. J. Am. Chem. Soc. 2008, 130, 10345-10353.

(5) Laage, D.; Stirnemann, G.; Hynes, J. T. Why Water Reorientation Slows Without Iceberg Formation Around Hydrophobic Solutes. J. Phys. Chem. B 2009, 113, 24282435.

(6) Hunger, J.; Tielrooij, K.-J.; Buchner, R.; Bonn, M.; Bakker, H. J. Complex Formation in Aqueous Trimethylamine-N-Oxide (TMAO) Solutions. J. Phys. Chem. B 2012, 116, $4783-4795$.

(7) Knake, L.; Schwaab, G.; Kartaschew, K.; Havenith, M. Solvation Dynamics of Trimethylamine N-Oxide in Aqueous Solution Probed by Terahertz Spectroscopy. J. Phys. Chem. B 2015, 119, 13842-13851.

(8) Comez, L.; Paolantoni, M.; Corezzi, S.; Lupi, L.; Sassi, P.; Morresi, A.; Fioretto, D. Aqueous Solvation of Amphiphilic Molecules by Extended Depolarized Light Scattering: The Case of Trimethylamine-N-Oxide. Phys. Chem. Chem. Phys. 2016, 18, 8881-8889.

(9) Ramakrishnan, G.; González-Jiménez, M.; Lapthorn, A. J.; Wynne, K. Spectrum of Slow and Super-Slow (Picosecond to Nanosecond) Water Dynamics Around Organic and Biological Solutes. J. Phys. Chem. Lett 2017, 8, 2964-2970.

(10) Tielrooij, K.-J.; Hunger, J.; Buchner, R.; Bonn, M.; Bakker, H. J. Influence of Concentration and Temperature on the Dynamics of Water in the Hydrophobic Hydration Shell of Tetramethylurea. J. Am. Chem. Soc. 2010, 132, 15671-15678. 
(11) Rossky, P. J.; Karplus, M. Solvation. a Molecular Dynamics Study of a Dipeptide in Water. J. Am. Chem. Soc. 1979, 101, 1913-1937.

(12) Titantah, J. T.; Karttunen, M. Long-Time Correlations and Hydrophobe-Modified Hydrogen-Bonding Dynamics in Hydrophobic Hydration. J. Am. Chem. Soc. 2012, $134,9362-9368$.

(13) Homsi Brandeburgo, W.; van der Post, S. T.; Meijer, E. J.; Ensing, B. On the Slowdown Mechanism of Water Dynamics Around Small Amphiphiles. Phys. Chem. Chem. Phys. 2015, 17, 24968-24977.

(14) Usui, K.; Hunger, J.; Sulpizi, M.; Ohto, T.; Bonn, M.; Nagata, Y. Ab Initio Liquid Water Dynamics in Aqueous TMAO Solution. J. Phys. Chem. B 2015, 119, 1059710606.

(15) Rezus, Y. L. A.; Bakker, H. J. Observation of Immobilized Water Molecules Around Hydrophobic Groups. Phys. Rev. Lett. 2007, 99, 148301.

(16) Silvestrelli, P. L. Are There Immobilized Water Molecules Around Hydrophobic Groups? Aqueous Solvation of Methanol from First Principles. J. Phys. Chem. B 2009, 113, 10728-10731.

(17) Titantah, J. T.; Karttunen, M. Water Dynamics: Relation Between Hydrogen Bond Bifurcations, Molecular Jumps, Local Density \& Hydrophobicity. Sci. Rep. 2013, 3, 2991.

(18) Sharp, K. A.; Madan, B.; Manas, E.; Vanderkooi, J. M. Water Structure Changes Induced by Hydrophobic and Polar Solutes Revealed by Simulations and Infrared Spectroscopy. J. Chem. Phys. 2001, 114, 1791.

(19) Imoto, S.; Forbert, H.; Marx, D. Water Structure and Solvation of Osmolytes at High 
Hydrostatic Pressure: Pure Water and TMAO Solutions at 10 kbar Versus 1 Bar. Phys. Chem. Chem. Phys. 2015, 17, 24224-24237.

(20) Ohto, T.; Hunger, J.; Backus, E. H. G.; Mizukami, W.; Bonn, M.; Nagata, Y. Trimethylamine-N-Oxide: Its Hydration Structure, Surface Activity, and Biological Function, Viewed by Vibrational Spectroscopy and Molecular Dynamics Simulations. Phys. Chem. Chem. Phys. 2017, 19, 6909-6920.

(21) Kast, K. M.; Brickmann, J.; Kast, S. M.; Berry, R. S. Binary Phases of Aliphatic NOxides and Water:? Force Field Development and Molecular Dynamics Simulation. J. Phys. Chem. A 2003, 107, 5342-5351.

(22) Paul, S.; Patey, G. N. Why Tert-Butyl Alcohol Associates in Aqueous Solution but Trimethylamine-N-Oxide Does Not. J. Phys. Chem. B 2006, 110, 10514-10518.

(23) Larini, L.; Shea, J.-E. Double Resolution Model for Studying TMAO/Water Effective Interactions. J. Phys. Chem. B 2013, 117, 13268-13277.

(24) Schneck, E.; Horinek, D.; Netz, R. R. Insight into the Molecular Mechanisms of Protein Stabilizing Osmolytes from Global Force-Field Variations. J. Phys. Chem. B 2013, 117, 8310-8321.

(25) Rodríguez-Ropero, F.; Rötzscher, P.; van der Vegt, N. F. A. Comparison of Different TMAO Force Fields and Their Impact on the Folding Equilibrium of a Hydrophobic Polymer. J. Phys. Chem. B 2016, 120, 8757-8767.

(26) Canchi, D. R.; Jayasimha, P.; Rau, D. C.; Makhatadze, G. I.; Garcia, A. E. Molecular Mechanism for the Preferential Exclusion of TMAO from Protein Surfaces. J. Phys. Chem. B 2012, 116, 12095-12104.

(27) Mondal, J.; Stirnemann, G.; Berne, B. J. When Does Trimethylamine N-Oxide Fold a Polymer Chain and Urea Unfold It? J. Phys. Chem. B 2013, 117, 8723-8732. 
(28) Mondal, J.; Halverson, D.; Li, I. T. S.; Stirnemann, G.; Walker, G. C.; Berne, B. J. How Osmolytes Influence Hydrophobic Polymer Conformations: A Unified View from Experiment and Theory. Proc. Natl. Acad. Sci. USA 2015, 112, 9270-9275.

(29) Athawale, M. V.; Dordick, J. S.; Garde, S. Osmolyte Trimethylamine-N-Oxide Does Not Affect the Strength of Hydrophobic Interactions: Origin of Osmolyte Compatibility. Biophys. J. 2005, 89, 858-866.

(30) Kokubo, H.; Hu, C. Y.; Pettitt, B. M. Peptide Conformational Preferences in Osmolyte Solutions: Transfer Free Energies of Decaalanine. J. Am. Chem. Soc. 2011, 133, 18491858.

(31) Panuszko, A.; Bruździak, P.; Zielkiewicz, J.; Wyrzykowski, D.; Stangret, J. Effects of Urea and Trimethylamine-N-Oxide on the Properties of Water and the Secondary Structure of Hen Egg White Lysozyme. J. Phys. Chem. B 2009, 113, 14797-14809.

(32) Canchi, D. R.; García, A. E. Cosolvent Effects on Protein Stability. Annu. Rev. Phys. Chem. 2013, 64, 273-293.

(33) Zou, Q.; Bennion, B. J.; Daggett, V.; Murphy, K. P. The Molecular Mechanism of Stabilization of Proteins by TMAO and Its Ability to Counteract the Effects of Urea. J. Am. Chem. Soc. 2002, 124, 1192-1202.

(34) The CP2K Developers Group. Available at: http://www.cp2k.org/.

(35) VandeVondele, J.; Krack, M.; Mohamed, F.; Parrinello, M.; Chassaing, T.; Hutter, J. Quickstep: Fast and Accurate Density Functional Calculations Using a Mixed Gaussian and Plane Waves Approach. Comput. Phys. Comm. 2005, 167, 103 - 128.

(36) Lippert, G.; Hutter, J.; Parrinello, M. A Hybrid Gaussian and Plane Wave Density Functional Scheme. Mol. Phys. 1997, 92, 477-488. 
(37) Jonchiere, R.; Seitsonen, A. P.; Ferlat, G.; Saitta, A. M.; Vuilleumier, R. Van Der Waals Effects in Ab Initio Water at Ambient and Supercritical Conditions. J. Chem. Phys. 2011, 135, 154503.

(38) Becke, A. D. Density-Functional Exchange-Energy Approximation with Correct Asymptotic Behavior. Phys. Rev. A 1988, 38, 3098-3100.

(39) Lee, C.; Yang, W.; Parr, R. G. Development of the Colle-Salvetti Correlation-Energy Formula into a Functional of the Electron Density. Phys. Rev. B 1988, 37, 785-789.

(40) Grimme, S.; Antony, J.; Ehrlich, S.; Krieg, H. A Consistent and Accurate Ab Initio Parametrization of Density Functional Dispersion Correction (DFT-D) for the 94 Elements H-Pu. J. Chem. Phys. 2010, 132, 154104.

(41) Goedecker, S.; Teter, M.; J, H. Separable Dual-Space Gaussian Pseudopotentials. Phys. Rev. B 1996, 54, 1703-1710.

(42) Berendsen, H. J. C.; Grigera, J. R.; Straatsma, T. P. The Missing Term in Effective Pair Potentials. J. Phys. Chem. 1987, 91, 6269-6271.

(43) Laage, D.; Stirnemann, G.; Sterpone, F.; Rey, R.; Hynes, J. T. Reorientation and Allied Dynamics in Water and Aqueous Solutions. Annu. Rev. Phys. Chem. 2011, 62, $395-416$.

(44) Laage, D.; Hynes, J. T. On the Molecular Mechanism of Water Reorientation. J. Phys. Chem. B 2008, 112, 14230-14242.

(45) Yoo, S.; Xantheas, S. S. Communication: The Effect of Dispersion Corrections on the Melting Temperature of Liquid Water. J. Chem. Phys. 2011, 134, 121105.

(46) Lee, H.-S.; Tuckerman, M. E. Dynamical Properties of Liquid Water from Ab Initio Molecular Dynamics Performed in the Complete Basis Set Limit. J. Chem. Phys. 2007, $126,164501$. 
(47) Grossman, J. C.; Schwegler, E.; Draeger, E. W.; Gygi, F.; Galli, G. Towards an Assessment of the Accuracy of Density Functional Theory for First Principles Simulations of Water. J. Chem. Phys. 2004, 120, 300-311.

(48) Schwegler, E.; Grossman, J. C.; Gygi, F.; Galli, G. Towards an Assessment of the Accuracy of Density Functional Theory for First Principles Simulations of Water. II. J. Chem. Phys. 2004, 121, 5400-5409.

(49) Rezus, Y. L. A.; Bakker, H. J. On the Orientational Relaxation of HDO in Liquid Water. J. Chem. Phys. 2005, 123, 114502.

(50) Morawietz, T.; Singraber, A.; Dellago, C.; Behler, J. How Van Der Waals Interactions Determine the Unique Properties of Water. Proc. Natl. Acad. Sci. USA 2016, 113, $8368-8373$.

(51) Rossato, L.; Rossetto, F.; Silvestrelli, P. L. Aqueous Solvation of Methane from First Principles. J. Phys. Chem. B 2012, 116, 4552-4560.

(52) Stirnemann, G.; Hynes, J. T.; Laage, D. Water Hydrogen Bond Dynamics in Aqueous Solutions of Amphiphiles. J. Phys. Chem. B 2010, 114, 3052-3059.

(53) Duboué-Dijon, E.; Fogarty, A. C.; Laage, D. Temperature Dependence of Hydrophobic Hydration Dynamics: From Retardation to Acceleration. J. Phys. Chem. B 2014, 118, $1574-1583$.

(54) Godec, A.; Merzel, F. Physical Origin Underlying the Entropy Loss upon Hydrophobic Hydration. J. Am. Chem. Soc 2012, 134, 17574-17581.

(55) Rezus, Y. L. A.; Bakker, H. J. Strong Slowing down of Water Reorientation in Mixtures of Water and Tetramethylurea. J. Phys. Chem. A 2008, 112, 2355-2361.

(56) Laage, D.; Hynes, J. T. A Molecular Jump Mechanism of Water Reorientation. Science 2006, 311, 832-835. 
(57) Stirnemann, G.; Rossky, P. J.; Hynes, J. T.; Laage, D. Water Reorientation, HydrogenBond Dynamics and 2D-IR Spectroscopy Next to an Extended Hydrophobic Surface. Faraday discuss. 2010, 146, 263-281.

(58) Stirnemann, G.; Sterpone, F.; Laage, D. Dynamics of Water in Concentrated Solutions of Amphiphiles: Key Roles of Local Structure and Aggregation. J. Phys. Chem. B 2011, 115, 3254-3262.

(59) Fogarty, A. C.; Duboué-Dijon, E.; Laage, D.; Thompson, W. H. Origins of the NonExponential Reorientation Dynamics of Nanoconfined Water. J. Chem. Phys 2014, $141,18 \mathrm{C} 523$.

(60) Fogarty, A. C.; Coudert, F. X.; Boutin, A.; Laage, D. Reorientational Dynamics of Water Confined in Zeolites. ChemPhysChem 2014, 15, 521-529.

(61) Boisson, J.; Stirnemann, G.; Laage, D.; Hynes, J. T. Water Reorientation Dynamics in the First Hydration Shells of F- and I-. Phys. Chem. Chem. Phys. 2011, 13, 19895.

(62) Stirnemann, G.; Wernersson, E.; Jungwirth, P.; Laage, D. Mechanisms of Acceleration and Retardation of Water Dynamics by Ions. J. Am. Chem. Soc. 2013, 135, 1182411831.

(63) Sterpone, F.; Stirnemann, G.; Laage, D. Magnitude and Molecular Origin of Water Slowdown Next to a Protein. J. Am. Chem. Soc. 2012, 134, 4116-4119.

(64) Sterpone, F.; Stirnemann, G.; Hynes, J. T.; Laage, D. Water Hydrogen-Bond Dynamics Around Amino Acids: The Key Role of Hydrophilic Hydrogen-Bond Acceptor Groups. J. Phys. Chem. B 2010, 114, 2083-2089.

(65) Stirnemann, G.; Laage, D. Communication: On the Origin of the Non-Arrhenius Behavior in Water Reorientation Dynamics. J. Chem. Phys. 2012, 137, 031101. 
(66) Galamba, N. Water Tetrahedrons, Hydrogen-Bond Dynamics, and the Orientational Mobility of Water Around Hydrophobic Solutes. J. Phys. Chem. B 2014, 118, 41694176.

(67) Usui, K.; Nagata, Y.; Hunger, J.; Bonn, M.; Sulpizi, M. A New Force Field Including Charge Directionality for TMAO in Aqueous Solution. J. Chem. Phys. 2016, 145, 064103.

(68) Meersman, F.; Bowron, D.; Soper, A. K.; Koch, M. H. J. Counteraction of Urea by Trimethylamine N-Oxide Is Due to Direct Interaction. Biophys. J. 2009, 97, 25592566.

(69) Bakulin, A. A.; Pshenichnikov, M. S.; Bakker, H. J.; Petersen, C. Hydrophobic Molecules Slow Down the Hydrogen-Bond Dynamics of Water. J Phys Chem A 2011, $115,1821-1829$.

(70) Bakulin, A. A.; Liang, C.; la Cour Jansen, T.; Wiersma, D. A.; Bakker, H. J.; Pshenichnikov, M. S. Hydrophobic solvation: a 2D IR spectroscopic inquest. Acc Chem Res 2009, 42, 1229-38.

(71) Wilkins, D. M.; Manolopoulos, D. E.; Pipolo, S.; Laage, D.; Hynes, J. T. Nuclear Quantum Effects in Water Reorientation and Hydrogen-Bond Dynamics. J. Phys. Chem. Lett 2017, 2602-2607. 


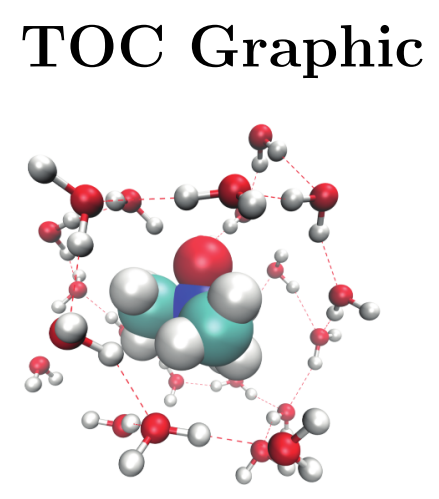

